\title{
Mobilizing the fountain of youth within
}

\author{
H. William Strauss, MD, and Josef J. Fox, MD
}

\section{See related article, pp. 463-471}

Toyama et $\mathrm{al}^{1}$ describe the favorable outcome of a 14-day course of granulocyte colony-stimulating factor (G-CSF) in patients, mean age 68, with chronic symptomatic ischemic heart disease who were not candidates for revascularization. Patients were randomized to either treatment with $1.5 \mu \mathrm{g} / \mathrm{kg}$ of G-CSF subcutaneously for 14 consecutive days $(n=20)$ or a saline control $(n=20)$. Prior to treatment G-CSF patients and controls had stress myocardial scintigraphy (exercise stress in 13 G-CSF and 12 control patients and ATP pharmacologic stress in 7 G-CSF and 8 control subjects). All subjects were assessed with cardiopulmonary exercise tests and Canadian Cardiovascular Society score (categorizing effort related angina). In addition, the investigators measured serial white blood cell (WBC) counts, brain natriuretic peptide (BNP), C-reactive protein (CRP), IL-6, and creatinine. The tests were repeated 3 months post-treatment. The total defect score of the stress perfusion abnormality in the G-CSF-treated group decreased from $20.4 \pm 11.2$ to $13.6 \pm 11.0$, while the control group defect score was essentially unchanged $21.7 \pm 10.9$ to $21.6 \pm 10.5$. LVEF and anaerobic threshold were unchanged, but peak $\mathrm{VO}_{2}$ increased slightly in the G-CSF group. This improvement was also reflected by a significant decrease in the Canadian Cardiovascular Society score in the G-CSF group. There were no significant changes in BNP, CRP, or IL-6. As anticipated, the WBC count increased from $\sim 5,000$ at baseline to $\sim 20,000$ by the fourth day of treatment. There were no adverse events in the treatment or control subjects during the 3-month interval of observation.

Endogenous G-CSF, a 175 amino acid $18.8 \mathrm{kD}$ protein, stimulates neutrophil progenitor cell proliferation in

From the Section of Nuclear Medicine, Memorial Sloan Kettering Cancer Center, New York, NY.

Reprint requests: H. William Strauss, MD, Section of Nuclear Medicine, Memorial Sloan Kettering Cancer Center, Room S212E, 1275

York Avenue, New York, NY 10065; straussh@mskcc.org.

J Nucl Cardiol 2011;18:396-7.

$1071-3581 / \$ 34.00$

Copyright $(\subset 2011$ American Society of Nuclear Cardiology.

doi:10.1007/s12350-011-9363-2 the bone marrow. G-CSF is produced by monocytes, macrophages, lymphocytes, fibroblasts, bone marrow stromal cells, and endothelial cells. Although endogenous G-CSF is glycosylated, the carbohydrate moiety is not required for biologic activity. ${ }^{2} \mathrm{G}-\mathrm{CSF}$ is used clinically to increase the WBC count in patients with neutropenia. In contrast to the physiologic glycosylated protein, the therapeutic drug approved in the US, filgrastim, manufactured by recombinant DNA technology, is not glycosylated. G-CSF binds to its specific cell-surface receptor, granulocyte colony-stimulating factor receptor (G-CSF-R). In the bone marrow, the interaction of G-CSF with G-CSF-R stimulates: hematopoietic stem cell proliferation, differentiation, commitment, and priming of some end-cell functional activation (such as phagocytic ability, respiratory burst metabolism, and antibody dependent killing). ${ }^{3}$ The effects of colony-stimulating factors on stem cells appear to be dose- and, possibly, duration-dependent. The WBC count typically returns to baseline levels within 4 days of discontinuing filgrastim. In addition to hematopoietic tissue, G-CSF receptors have also been identified on cardiomyocytes, neuronal precursors, endothelial cells, and placental tissue. ${ }^{4} \mathrm{G}-\mathrm{CSF} / \mathrm{G}-$ CSF-R interaction in these non-hematopoietic tissues stimulates the proliferation, migration, and differentiation of progenitor cells, and also inhibits apoptosis. ${ }^{5}$

In patients with acute cardiac syndromes, there is a physiologic increase in CD34 endothelial progenitor cells in the circulation, reaching a peak of about 5.8 times baseline at about 7 days following the acute event. The importance of these progenitor cells in reducing or reversing the acute injury is uncertain. For the progenitor cells to achieve repair/regeneration of injured tissue, the cells must find their way to the site of injury, engraft the site of injury, and stimulate repair of the injured tissue. Homing requires expression of membrane receptors on the progenitor cells (such as CXCR-4, CD117, hepatocyte growth factor, ICAM, and/or VCAM). These receptors on the progenitor cells recognize chemokines (SDF-1, SCF, lymphocyte function associated antigen-1 (LFA-1), and/or very late antigen-4 (VLA-4)) which are expressed in the border zone of infarction. When the progenitor cells localize at the site of injury, they provide a paracrine function, with secretion of VEGF, FGF, SDF-1, as well as factors preventing apoptosis of cardiomyocytes in the periinfarct zone. The bone marrow stem cells also stimulate 
the mobilization of resident cardiac progenitor cells to help heal the injury. In normal patients $<0.001 \%$ of their circulating white cells express CD34. Following G-CSF, this number may increase 2 to 10 fold.

Shim et al $^{6}$ reviewed 13 studies that utilized G-CSF as a therapeutic agent in patients with acute coronary syndromes. G-CSF was administered at various times following injury, ranging from immediately after rescue angioplasty to several days following the event-without a clear indication of benefit of either early or late therapy. There were also differences in administered dose, generally ranging from $5 \mu \mathrm{g} / \mathrm{kg}$ as a single dose to $10 \mu \mathrm{g} / \mathrm{kg}$ administered daily for 6 days, again, with no clear indication of beneficial effects of low-dose or highdose administration. There was, however, an increased incidence of restenosis and vascular occlusive events in patients treated with higher dose therapy. 6,7

Although the use of G-CSF to repair/regenerate myocardial tissue is not new, the publication by Toyama and associates has several novel features. Previous studies have generally used G-CSF in patients following an acute coronary event. However, small groups of patients with chronic ischemia have been treated with brief courses of $\mathrm{G}-\mathrm{CSF}^{8}$, with some success. In one previous study, Suzuki et $\mathrm{al}^{7}$ tested the role of a different form of recombinant G-CSF, lenograstim ${ }^{1}$, at a dose of $3.1 \mu \mathrm{g} / \mathrm{kg} /$ day subcutaneously for 10 days in two groups of patients: (a) following acute infarction due to LAD occlusion, and (b) patients with chronic ischemic heart disease. Serial myocardial perfusion studies in both the groups of patients treated with G-CSF demonstrated a significant decrease in the extent of ischemia. In the current study, Toyama et $\mathrm{al}^{1}$ document a beneficial effect with filgrastim (known to be about $25 \%$ less potent than lenograstim ${ }^{7}$ ) in patients with severe but stable chronic ischemic heart disease, at a lower dose of G-CSF $(1.5 \mu \mathrm{g} / \mathrm{kg})$ administered over a longer interval of time (14 days).
The current study by Toyama et $\mathrm{al}^{1}$ confirms the earlier work of Suzuki, demonstrating an objective, beneficial effect of G-CSF therapy in a population patients with chronic ischemic heart disease who are not candidates for revascularization. More studies are needed to determine whether the major beneficial effects are due to a direct effect of G-CSF on cardiomyocytes (or resident cardiac stem cells), or from the mobilization of bone marrow progenitor cells. The answer to that question may help define the most favorable combination of the dose of G-CSF and duration of therapy.

\section{References}

1. Toyama T, Hoshizaki H, Kasama S, Yamashita E, Kawaguchi R, Adachi H, Oshima S, Hasegawa A, Kurabayashi M. Low-dose and long-term G-CSF treatment can improve severe myocardial ischemia in patients with severe coronary artery disease. J Nucl Cardiol 2011. doi:10.1007/s12350-011-9350-7.

2. Metcalf D. The Florey lecture, 1991: The colony stimulating factors: Discovery to clinical use. Philos Trans Biol Sci 1991; 333:147-73.

3. Filgrastim package insert: http://www.neupogen.com/pdf/Neupogen_ PI.pdf.

4. Liongue C, Wright C, Russell AP, Ward AC. Granulocyte colony stimulating factor receptor: Stimulating granulopoiesis and much more. Int J Biochem Cell Biol 2009;41:2372-5.

5. Brunner S, Engelman MG, Franz WM. Stem cell mobilization for myocardial repair. Exp Opin Biol Ther 2008;8:1675-90.

6. Shim W, Mehta A, Lim SY, Zhang G, Lim CH, Chua T, et al. G-CSF for stem cell therapy in acute myocardial infarction: Friend or foe? Cardiovasc Res 2011;89:20-30.

7. Suzuki K, Nagashima K, Arai M, Uno Y, Misao Y, Takemura G, et al. Effect of granulocyte stimulating factor treatment at a low dose but for a long duration in patients with coronary heart disease. Circ J 2006;70:430-7.

8. Kastrup J, Ripa RS, Wang Y, Jorgensen E. Myocardial regeneration induced by granulocyte colony stimulating factor mobilization of stem cells in patients with acute or chronic ischaemic heart disease: A non-invasive alternative for clinical stem cell therapy? Eur Heart J 2006;27:2748-54.

\footnotetext{
${ }^{1}$ Lenograstim is a glycosylated G-CSF, produced by recombinant DNA technology. The agent is available in Europe and Japan.
} 